\section{CENTRO DE EDUCACION ESPECIAL POLIVALENTE TENERIFE/ESPAÑA}

\author{
Rubens Henriquez Hernández, \\ Arquitecto \\ $141-94$
}

\section{SINOPSIS}

A partir de las recomendaciones de la Organización Mundial de la Salud, sobre los requisitos que deben reunir los centros que se encargan de la atención de los niños subnormales, se ha realizado este conjunto con capacidad para unos 400 de estos niños.

Entre su gran diversidad de instalaciones cuenta con secciones de diagnóstico y terapia, servicios directivos, administrativos y de asistencia social con unidades de habitación, trabajo, formación, recreo y deporte.

Como notas más destacables del mismo pueden mencionarse: la clara diferenciación de funciones en bloques independientes, con el fin de centralizar los usos y reducir los volúmenes necesarios para hacerlos más asequibles a la escala de los niños; la adaptación al fuerte desnivel del terreno, con una disposición escalonada que posibilita mejores vistas sobre el mar cercano, y la economía estructural, debida a su modulación, que permitió invertir el ahorro en mejores acabados y tratamientos.
Cada vez se da más importancia a la atención de los niños que necesitan un cuidado especial para su desarrollo. La Organización Mundial de la Salud ha realizado una serie de estudios, reuniones, publicaciones, con los que ha sentado principios que afectan tanto a las actividades de la enseñanza especial que necesitan estos niños, como a la arquitectura de los centros donde ha de impartirse. La aplicacion de estos principios requiere contar con personal, clases, centros y servicios especiales, adecuados a las circunstancias de los niños y al ambiente que los rodea, a fin de establecer un sistema formativo que encauce al niño hacia su ajuste futuro en el medio social.

Para la realización del Centro se contaba con una parcela de $30.000 \mathrm{~m}^{2}$ que, además de permitir las instalaciones necesarias para dar cabida a $400 \mathrm{ni}-$ ños, preveía un espacio adicional para futuras ampliaciones, dado el carácter continuamente evolutivo de este tipo de enseñanza.

Se partio de un programa, redactado por la Direccion General de Enseñanza Primaria, a través de su seccion de Enseñanza Especial, que fue esquematizado desde un punto de vista funcional, agrupando las actividades fundamentales para conseguir una estructuración más orientativa. Dicho programa se ha seguido exactamente en el proyecto, si bien en algunos detalles se han sugerido innovaciones, discutidas en el Congreso de Siquiatria Infantil recientemente celebrado, en el que se expuso el anteproyecto preparado para atender el programa. Entre estos detalles cabe mencionar: la conveniencia de que el comedor fuera divisible; el

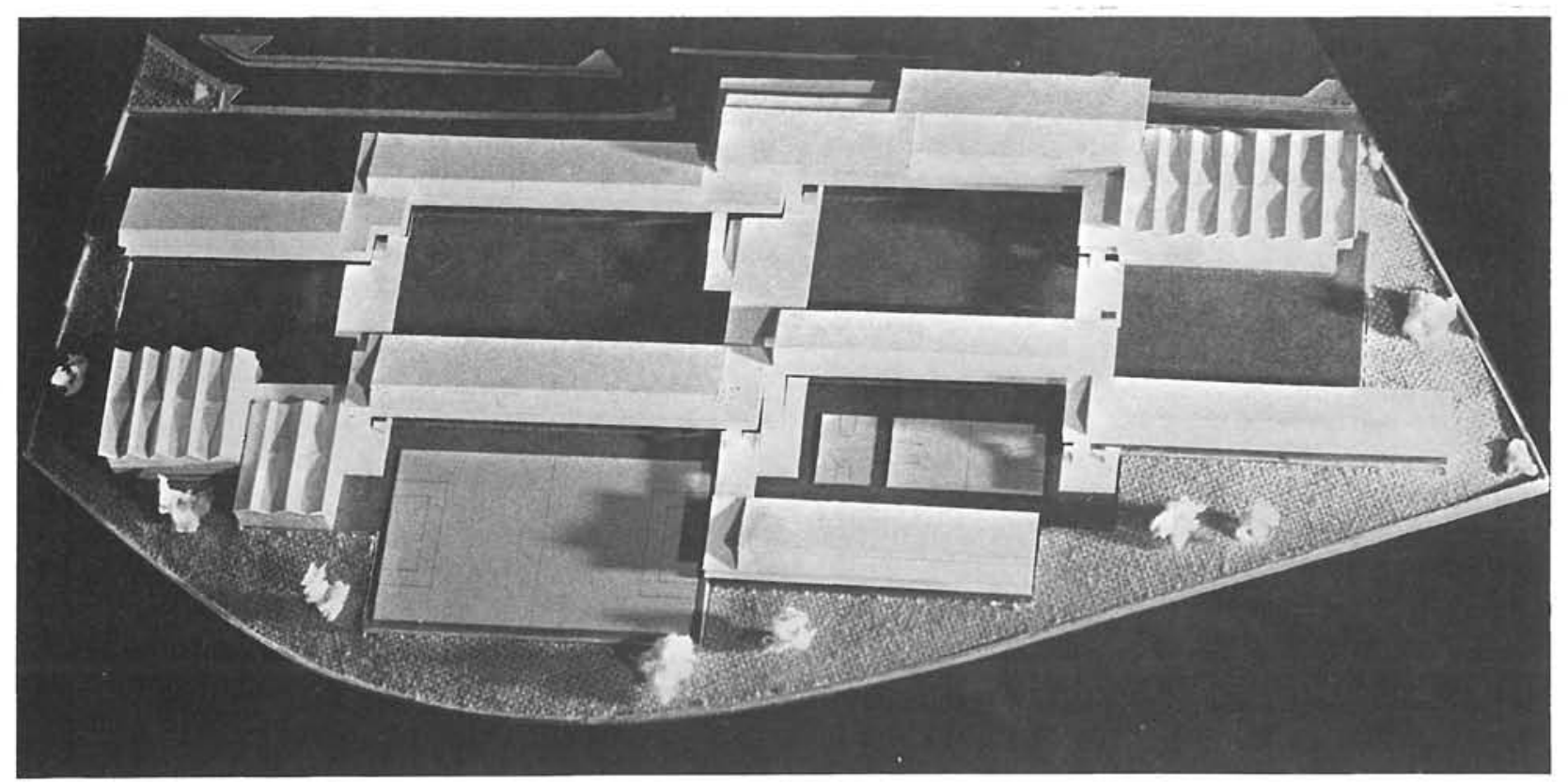




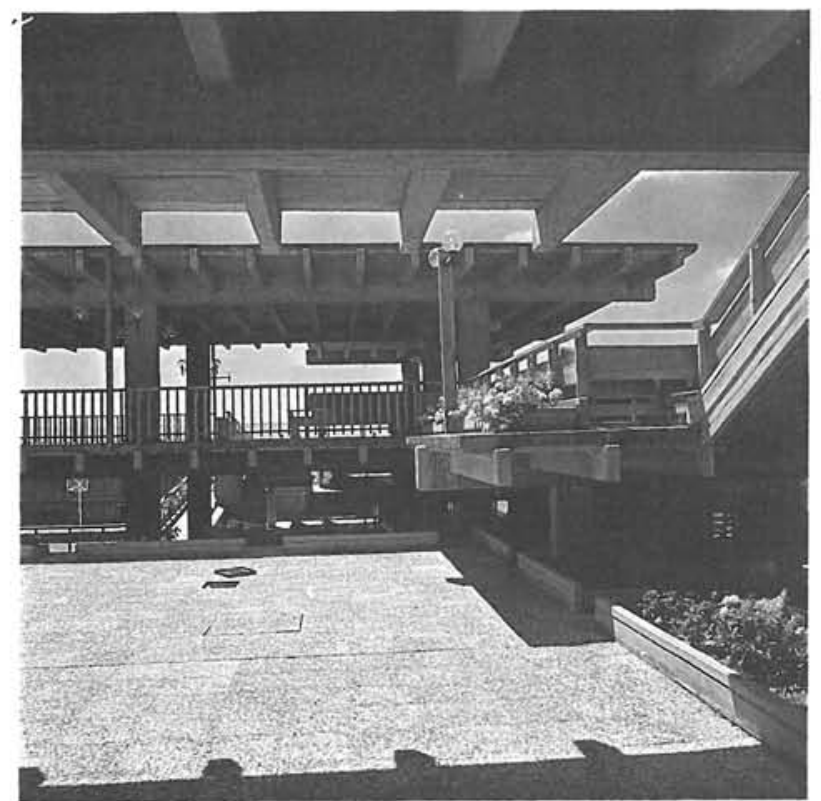

establecimiento de una unidad de enfermería conjunta, capaz de alojar del 5 al 10 por 100 de los internos, excluyéndola de los hogares, ya que asi el niño puede estar más atendido con un minimo de personal y se respeta más su ritmo normal de vida; disponer de un centro comunitario como complemento de los hogares; dar clases al aire libre, en unas terrazas prolongación de las aulas y agrupar dos hogares con un solo vigilante.

Además de la indicada sujeción al Programa, el proyecto responde a una serie de criterios sobre la mision de este tipo de centros: diagnostico, terapéutica en sus vertientes médica, sicológica, pedagógica y de orientación profesional; y, como meta final, la integracion social del deficiente, al que hay que estimular e inducir a ocupar ese sitio para el que se le ha procurado hacer apto. Hay que añadir el equipo administrativo, el directivo y la asistencia social.

El programa también establece que los centros de educacion especial deben ser organizaciones abiertas, de tipo familiar, en orden a la adaptación del subnormal, ya que el centro no es un fin sino un medio.

Partiendo de los principios generales enunciados, el proyecto tendio a acomodar los servicios fundamentales en edificios independientes, con el fin de reducir las dimensiones de éstos y conferir al conjunto un carácter más humano, procurando agrupar los edificios en colectividades alrededor de centros funcionales. Así mismo se articulo la planificacion de modo que se creara un conjunto variado de espacios y ambientes, poniendo especial cuidado en crear suficiente riqueza de volúmenes en la edificación para que el niño tuviera diversidades de vistas y sensación de movimiento. También se tuvo en cuenta que el programa de necesidad variaría probablemente al funcionar el

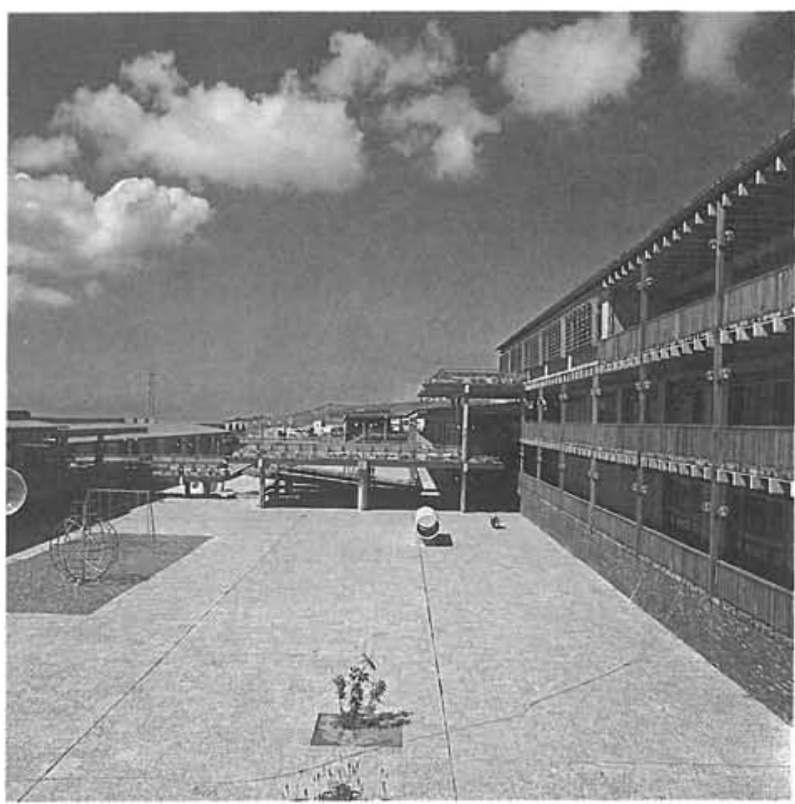

Centro, por lo que se preparo la zona de actividades sociales para poder sufrir dichas modificaciones.

Dado que la capacidad de relación social del niño subnormal es pequeña, era aconsejable organizar los internados en unidades familiares, diferenciando las unidades de habitacion, trabajo, formacion, recreo, tratamiento y asistencia médica; dirección, administración y relación con el exterior y servicio, intentando conseguir un equilibrio optimo entre nucleacion, para eficacia terapética, y centralización, para eficiencia mecánica. También se dispusieron centros monobloques en los que se agrupan unidades arquitectónicas que tienen una personalidad acusada, beneficiándose de la posibilidad de centralizar los servicios gracias a su concepcion vertical.

El esquema general de organización diferencia las aulas y talleres, los dormitorios, los deportes cubiertos y los servicios generales en un conjunto de edificios aislados, donde se agrupan funciones diferentes en distintas plantas, estructurados entre unos ambientes abiertos - formados por patios o espacios para deportes al aire libre- entre los distintos bloques. Estos se colocan en una misma direccion dada la pendiente del terreno, formando un escalonamiento de alturas equivalentes a medias plantas, que permite, desde los niveles altos de los edificios, la vista hacia el mar en el horizonte.

La volumetría general establece, en todas estas dependencias, un movimiento de volúmenes que dan variedad al conjunto dentro de una trama estructural múltiplo de $0,90 \mathrm{~m}$, formando modulos de $6,30 \times 6,30 \mathrm{~m}$, tanto para los espacios descubiertos como para los cuerpos de edificacion, que se diferencian exteriormente por sus distintos acabados. 


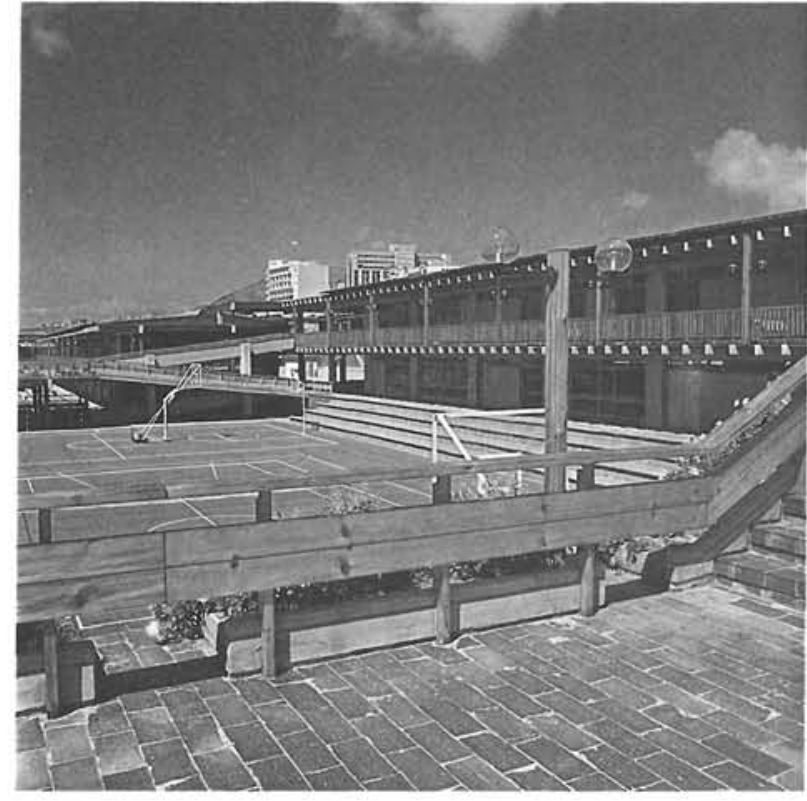

La entrada principal se sitúa en la parte más alta del terreno, en la calle más importante de las tres que rodean el conjunto y dando acceso a un aparcamiento que, por su disposicion con relacion al desnivel del terreno, permite la ventilación del bla que colindante.

El frente de este edificio, retranqueado de la calle por un jardin (que se interrumpe en su parte central por un paso-estacionamiento para los vehículos que llegan a la entrada), dispone de un vestibulo para los niños que, desde aqui, pueden distribuirse a los demás edificios del Centro a través de los pasillos cubiertos.

Ya en el interior cuenta con un gran vestíbulo que distribuye, a un lado, las secciones de administracion, asistencia social, direccion, secretaria, oficinas, salas de reunion y servicios, y, al otro, los servicios médicos y de reconocimiento.

En su planta inferior se disponen los comedores de profesores y alumnos, la sala de estar-cafetería y la cocina con todas sus dependencias auxiliares. Al otro lado del vestíbulo de esta planta se encuentran los almacenes de materiales de juego y los talleres, que tienen acceso independiente desde la calle y están comunicados directamente con los pasillos de circunvalación del Centro. En esta misma zona está la enfermería, en contacto con los servicios centrales.

En la parte alta, a nivel de la planta principal, se sitúan la capilla y el salon de actos, este último de dos alturas, diferenciado exteriormente por su cubierta.

En una tercera planta, que ocupa solo parte de la edificacion, se disponen los gabinetes de trabajo y seminarios, asi como clases grandes y la biblio teca técnica.

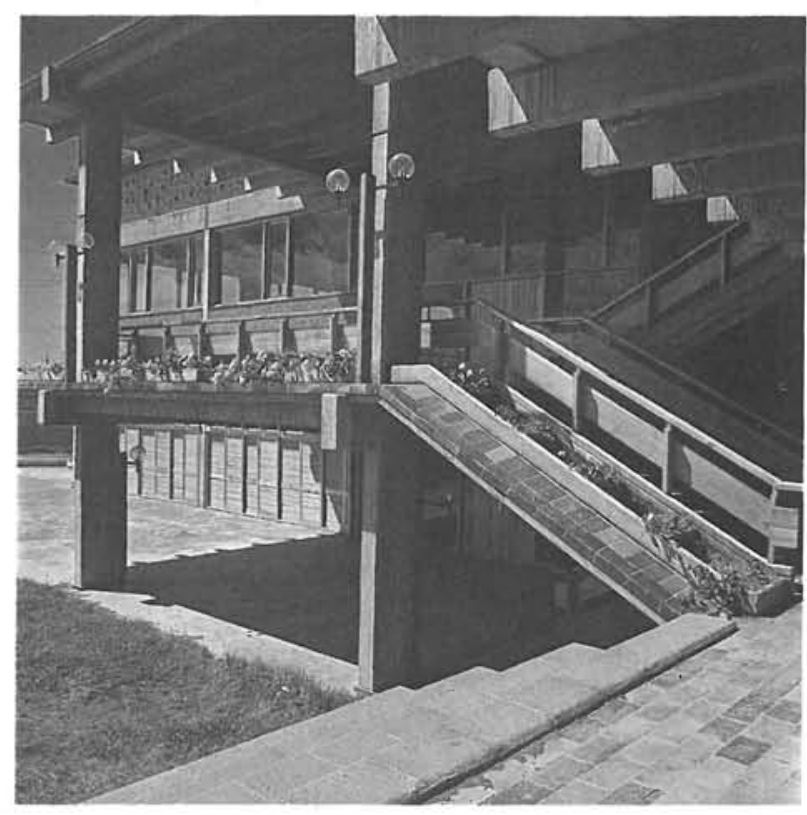

Este bloque cuenta con porches exteriores junto a los vestíbulos $y$, en planta baja, con una galería que conduce al comedor, dando frente a un gran patio que tiene a sus lados espacios asimismo descubiertos, visibles los unos desde los otros a traves de pasillos porticados que sirven de paso y estancia de los niños. Estos cuentan también con las galerias cubiertas formadas por los porches de los otros edificios que dan a los patios.

Los edificios de enseñanza, formados por dos bloques separados, agrupan las aulas en los distintos tamaños exigidos por el programa. Dichas aulas tienen pasillos de acceso por poniente $y$ unas amplias terrazas limitadas por vegetación en el lado contrario, que sirven como prolongación del aula y como transicion del ambiente interior hacia el espacio abierto exterior. Cada uno de estos blaques tiene un quiebro que deja en una parte las aulas mayores y en otra las menores. Debajo de una de estas partes están los talleres y debajo de la otra la biblioteca y las salas auxiliares.

Los internados se estructuran igualmente en dos bloques, que agrupan unidades familiares con tres dormitorios de cinco camas cada uno, una sala de estar central, aseos, un pequeño oficio y una habitacion doble para las cuidadoras, abierta a dos unidades familiares, lo que permite, con menos personal, atender a más niños. Estas unidades se disponen en dos plantas, con una galería exterior de acceso por el lado de levante.

Las instalaciones deportivas cubiertas comprenden un gimnasio, una piscina y sus instalaciones complementarias. Por su mayor altura y el tipo de cubierta, que permite grandes ámbitos interiores sin soportes intermedios, destacan del resto de las construcciones. En una planta baja se proyecta el salon destinado a club social y otras instalaciones. 


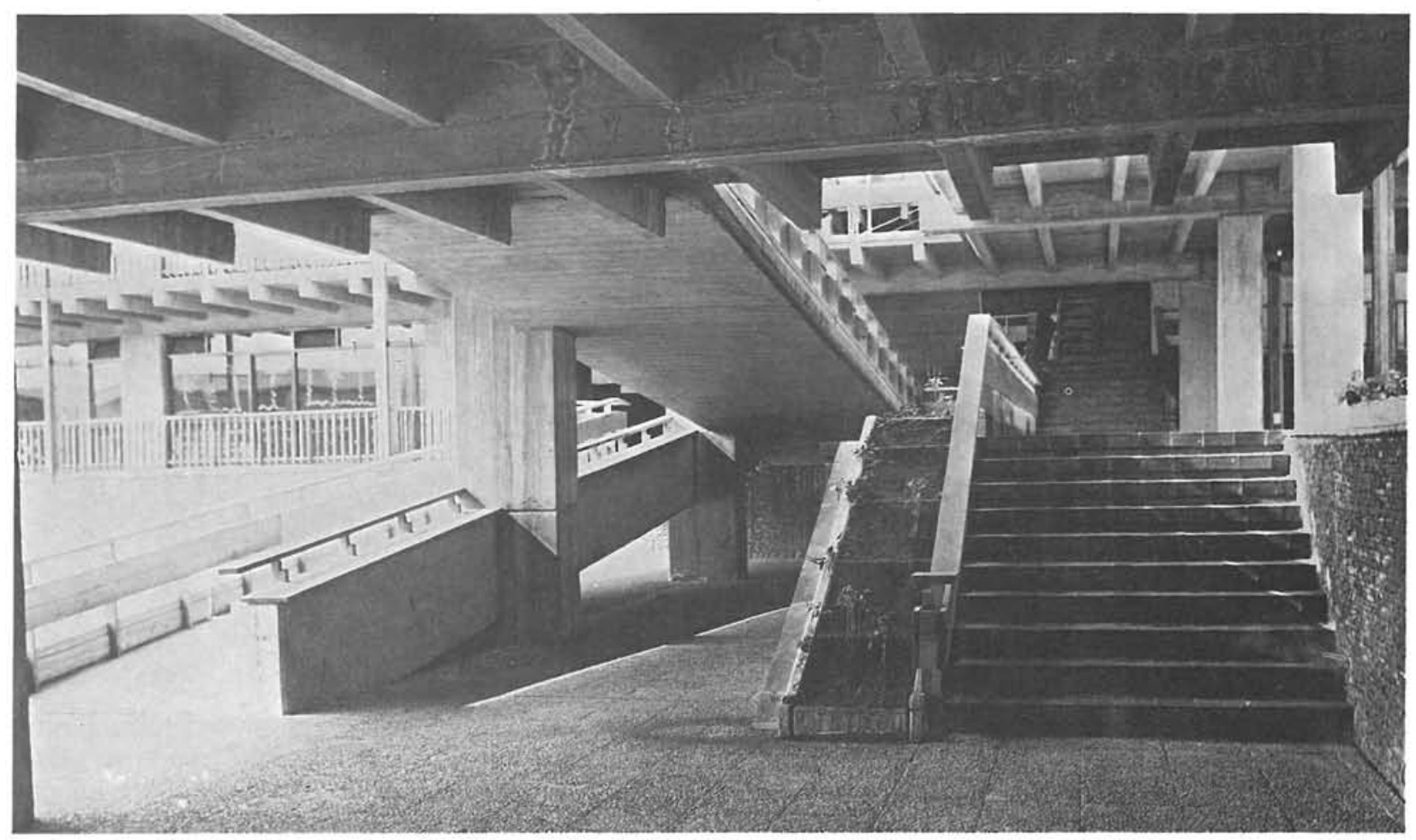

Los patios de juegos se disponen entre los edificios de servicios centrales y los de enseñanza y dormitorios, quedando articulados por pasos cubiertos y escaleras que salvan el escalonamiento del conjunto, el cual se mantiene con un modulo vertical uniforme de media planta. Los patios quedan en desnivel respecto a la planta baja, de manera que la galería inferior de la edificación da una cierta independencia del ambiente del patio.

Entre los segundos bloques de enseñanza y de dormitorios se sitúan los campos de deportes: un campo de fútbol con su graderío y una pista de baloncesto y otra de minibasket.

Como criterio general de organizacion se ha procurado que niños y niñas de edades mentales diferentes estén separados en las actividades docentes y en los hogares, pero que, al mismo tiempo, haya una serie de elementos comunes, como el

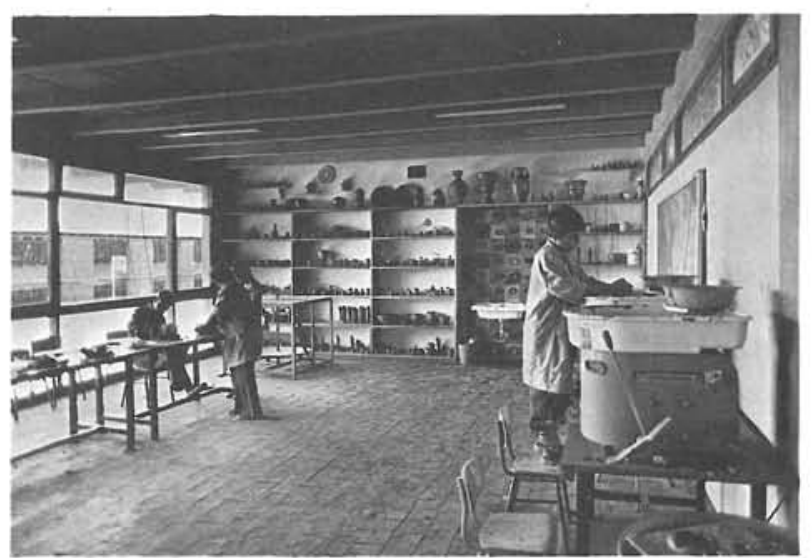

club social, el salon de actos, la biblioteca, el comedor y las zonas deportivas, en donde, en un momento dado, puedan estar agrupados.

La estructura general de las edificaciones es de hormigon armado visto, siguiendo la retícula general de modulacion, con un tipo de columnas y vigas, en sentido paralelo a la fachada, sobre la que apoyan nervios perpendiculares a ésta y que vuelan para constituir las galerías exteriores. EI ritmo del nervado confiere a la obra una cierta ambientación dentro de la arquitectura tradicional del país, a la que también contribuye el juego de patios y las galerías alargadas, a modo de balco nadas.

Con el fin de lograr una diferenciación dentro de la retícula modular que constituye la trama básica de los edificios, se ha jugado con la disposicion de galerías: en los bloques de aulas existen las

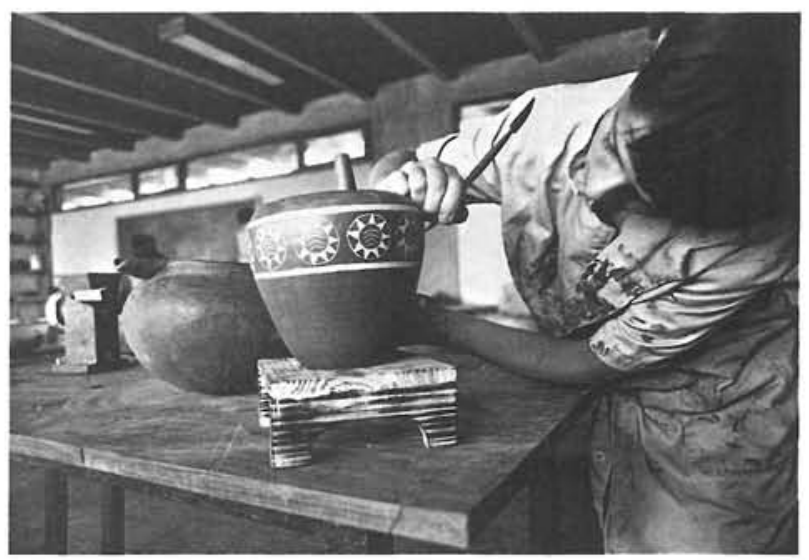


de acceso por un lado y las terrazas por el otro; en el cuerpo central prácticamente desaparecen, dando al edificio unos desplazamientos en sus distintas plantas de acuerdo con su programa de necesidades; los internados cuentan con galerias de acceso, y, en las zonas de dormitorios, vuelven a desaparecer, siendo aqui el elemento diferenciador los entrantes y salientes del muro de fachada. Se completa el acabado exterior general con las cubiertas del salon de actos y de los pabellones de deportes, en las que los forjados presentan un plegado que les permite salvar grandes luces.

Los materiales de revestimiento y acabado se han elegido en función de su duracion y facilidad de mantenimiento, disponiéndose en los paramentos de cada dependencia de acuerdo con su funcion. Entre ellos cabe destacar, por su profusión, la madera, utilizada en toda la carpinteria: en las paredes de la sala de estar y en todas las barandillas exteriores; la toba volcánica, que constituye todos los muros que aparecen en los múltiples desniveles; el terrazo, mármol y baldosa de cerámica prensada que cubren los suelos y los ele-

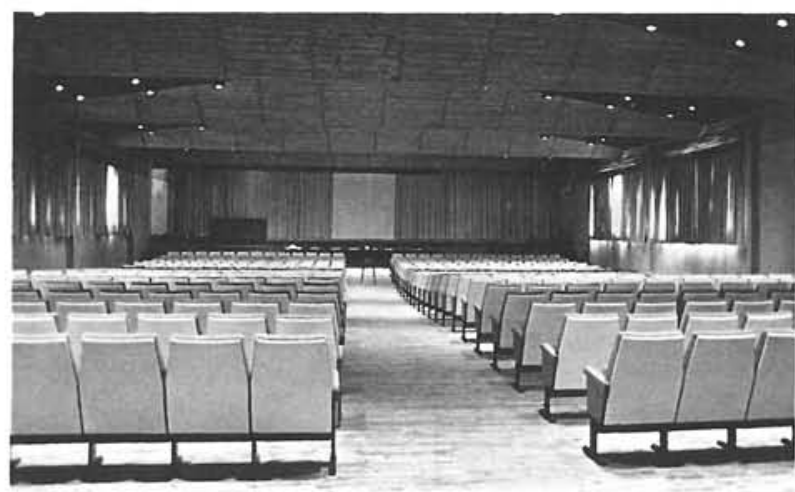

mentos prefabricados de pomez, utilizados en las compartimentaciones interiores.

Como gran elemento compositivo complementario de todos los edificios se utiliza la vegetación, que se prodiga en todo el Centro, con árboles en la zona de jardin perimetral que dan sombra donde los niños pueden guarecerse y otros arbustos y plantas que, con sus flores, producen un elemento de color agradable y vivo.

Adaptación de Obras y Proyectos: José Miguel Frutos

\section{publicación del i.e.t.c.c.}

\section{Vida, pensamiento y obra del genial arquitecto}

Las viviendas humanas - desde las más modestas hasta las mejor equipadas - han servido de base a los progresivos métodos de investigación clínica, con los que $\mathrm{Mr}$. J. Neutra ha pretendido descubrir la intimidad más recóndita de la vida humana. Los temas preferidos en sus estudios han sido las residencias infantiles, los jardines de la infancia, los sanatorios para niños, los centros de recreo juvenil $y$, en general, todas las variantes de los centros docentes. Siempre aplicó las formas a condiciones muy especificamente humanas, y siguió con apasionada atención los resultados que las distintas percepciones sensoriales motivaban en el sistema nervioso y endocrino de los hombres, de las mujeres y de los niños. Para Mr. Neutra, lo que cuenta, a la hora de la verdad, es el reflejo humano.

Un volumen encuadernado en tela de $21 \times 27,5 \mathrm{~cm}$, compuesto de 231 páginas y 217 fotos, dlbujos y flguras de linea.

Preclos: Espafia 2.000 ptas. Extranjero $\$ 29.00$.

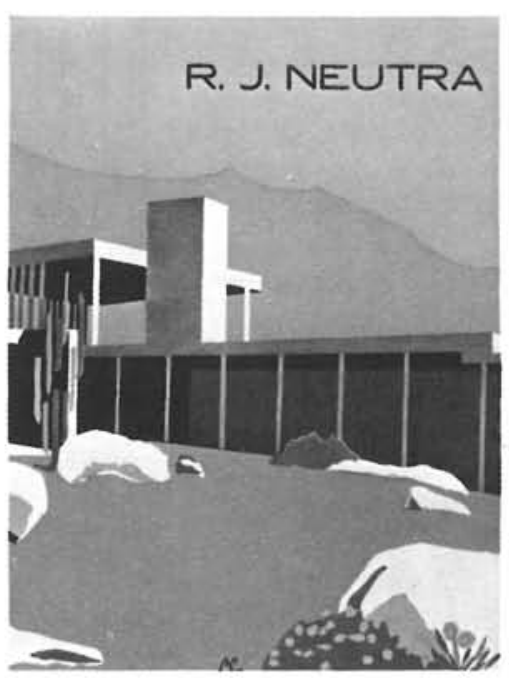

